\title{
Logic and bounded-width rational languages of posets over countable scattered linear orderings
}

\author{
Nicolas Bedon \\ Université Paris-Est and CNRS \\ Laboratoire d'informatique de l'Institut Gaspard Monge, UMR CNRS 8049 \\ 5, boulevard Descartes, Champs-sur-Marne \\ 77454 Marne-la-Vallée Cedex 2 \\ France \\ Nicolas.Bedon@univ-mlv.fr
}

\begin{abstract}
In this paper we consider languages of labelled $N$-free posets over countable and scattered linear orderings. We prove that a language of such posets is series-rational if and only if it is recognizable by a finite depth-nilpotent algebra if and only if it is bounded-width and monadic second-order definable. This extends previous results on languages of labelled $N$-free finite and $\omega$-posets and on languages of labelled countable and scattered linear orderings.
\end{abstract}

\section{Introduction}

It is known since the beginning of the 60's that automata, rational expressions, monadic second-order logic $(\mathrm{MSO}[<])$ and finite monoids all have the same expressive power for the definition of languages of finite words. They have been widely studied since that time, and adapted to more complex structures, like infinite words, trees, or partially ordered labelled sets. The subject of this paper is such an extension, from two different directions: languages of infinite words, and languages of posets. Let us cite the works it relies on.

First, Büchi $[6,7]$ initiated the study of automata on infinite words, in the meaning that letters are indexed by ordinal numbers, and not just integers as for the finite words case. He successfully used the equivalent expressive power of automata and $\mathrm{MSO}[<]$ in order to exhibit decision procedures for the decidability of this logic interpreted over ordinals. Extending the automata technique to trees, Rabin [18] studied the decidability of the monadic second-order theory of trees, from which he deduced decidability results on linear orders. Later, Bruyère and Carton [5] introduced automata and equivalent rational expressions for words whose shape is a countable and scattered linear ordering. These automata and rational expressions were recently connected to $\mathrm{MSO}[<]$ by Bedon, Bès, Carton and Rispal [2]. Adding the notion of parallelism to the notion of sequentiality in words, Lodaya and Weil [14] defined automata, rational expressions and finite algebra for languages of particular finite partially ordered sets, obtained using the letters and closure under sequential and parallel composition. This class of 
posets corresponds precisely to the class of $\mathrm{N}$-free posets $[22,23]$. The connection between the results of Lodaya and Weil and $\mathrm{MSO}[<]$ has been established by Kuske $[11,12]$, together with an extension to the infinite $(\omega)$ composition of Nfree finite posets. Finally, Bedon and Rispal [3] recently extended the Kleene-like theorems of Bruyère-Carton and Lodaya-Weil by defining automata and rational expressions for languages of $N$-free posets with a sequential composition indexed by a countable and scattered linear ordering.

This paper is a study of the connections between $\mathrm{MSO}[<]$, finite algebra and rational expressions for this class (denoted by $S P^{\diamond}$ ) of posets. We extend to $S P^{\diamond}$ the results of Kuske on $\mathrm{N}$-free $\omega$-posets and those of Bedon, Bès, Carton and Rispal on countable and scattered linear orderings. We prove that $\mathrm{MSO}[<]$, finite depth-nilpotent algebra and series-rational expressions have the same expressive power for bounded-width languages of posets of $S P^{\diamond}$. The logical formalism is due to Büchi, but interpreted over $S P^{\diamond}$ rather than well-ordered structures. The series-rational languages are those of Bruyère and Carton, extended with a commutative operation for finite parallel composition. Finally, the algebra are semigroups equipped with a sequential product adapted for sequences of elements indexed by scattered and countable linear orderings, and a commutative parallel composition of elements. Informally speaking, such an algebra is depth-nilpotent if any nested parallel composition leads to 0 beyond a fixed threshold. As the constructions are effective, this gives in particular a decision procedure that relies on automata theory for the theory of $\mathrm{MSO}[<]$ interpreted over boundedwidth posets of $S P^{\diamond}$. This decision result can also be obtained without automata theory, using for example model-theoretical techniques as in [20], or the methods of [13]. As a corollary, the inclusion problem of rational languages is also decidable.

The paper is organized as follows. Section 2 is devoted to basic definitions, linear orderings and posets. Sections 3, 4 and 5 respectively introduce seriesrational languages, algebra and logic for posets over scattered and countable linear orderings. The main result of the paper is stated in Section 6. It contains in particular sketches of constructions to obtain a finite depth-nilpotent algebra from a logical sentence, a logical sentence from a series-rational expression, and a series-rational expression from a finite depth-nilpotent algebra, which shows the equivalence between the three formalisms. Finally, Section 7 concludes.

\section{Basic definitions}

We start by some basic definitions on linear orderings. We refer to [19] for a survey on the subject. Let $J$ be a set equipped with an order $<$. The ordering $J$ is linear if all elements are comparable : for any distinct $j$ and $k$ in $J$, either $j<k$ or $k<j$. For any linear ordering $J$, we denote by $-J$ the backward linear ordering obtained from the set $J$ with the reverse ordering. A linear ordering $J$ is dense if for any $j$ and $k$ in $J$ such that $j<k$, there exists an element $i$ of $J$ such that $j<i<k$. It is scattered if it contains no dense sub-ordering. The ordering $\omega$ of natural integers is scattered. Ordinals are also scattered orderings. 
We denote by $\mathcal{N}$ the sub-class of finite linear orderings, $\mathcal{O}$ the class of countable ordinals and $\mathcal{S}$ the class of countable scattered linear orderings.

Definition 1. A linear ordering $J$ is complete if

- every non-empty sub-ordering $K$ of $J$ which is bounded above has a least upper bound in $J$, and

- every non-empty sub-ordering $K$ of $J$ which is bounded below has a greatest lower bound in $J$.

Example 1. Let $\alpha=\omega$ and $\beta=-\omega$. The scattered linear ordering $\gamma=\alpha+\beta$ is not complete, as the part of $\gamma$ composed of the elements of $\alpha$ (resp. $\beta$ ) does not respect the first (resp. last) condition of the definition. However, $\omega+1+-\omega$ is complete.

Let $J \in \mathcal{S}$ be a countable and scattered linear ordering. An interval $K$ of $J$ is a subset $K \subseteq J$ such that $\forall k_{1}, k_{2} \in K, \forall j \in J$, if $k_{1}<j<k_{2}$ then $j \in K$. A cut $(K, L)$ of $J$ is a partition of $J$ into two intervals $K$ and $L$ such that all the elements of $K$ are less than all the elements of $L$. Thus $J=K \cup L$ and $K \cap L=\emptyset$. The set of all cuts of $J$ is denoted by $\hat{J}=\{(K, L) \mid K \cup L=J \wedge \forall k \in K, \forall l \in$ $L, k<l\}$. The set $\hat{J}$ is naturally equipped with the ordering $\left(K_{1}, L_{1}\right)<\left(K_{2}, L_{2}\right)$ if and only if $K_{1} \subset K_{2}$. This linear ordering can be extended to $J \cup \hat{J}$ by setting $j<(K, L)$ whenever $j \in K$ for any $j \in J$ and $(K, L) \in \hat{J}$, and keeping the orderings on the elements of $J$ and of $\hat{J}$. We set $\hat{J}^{*}=\hat{J} \backslash\{(\emptyset, J),(J, \emptyset)\}$.

A poset $(P,<)$ is a set $P$ partially ordered by $<$. In order to lighten the notation we often denote the poset $(P,<)$ by $P$. An antichain is a subset $P^{\prime}$ of $P$ such that all elements of $P^{\prime}$ are incomparable (with $<$ ). The width of $P$ is

$$
w d(P)=\sup \{|E|: E \text { is an antichain of } P\}
$$

where sup denotes the least upper bound of the set. In this paper, we restrict to countable scattered posets which are thus partially ordered countable sets without any dense sub-ordering. Let $\left(P,<_{P}\right)$ and $\left(Q,<_{Q}\right)$ be two disjoint posets. The parallel composition of $\left(P,<_{P}\right)$ and $\left(Q,<_{Q}\right)$ is the poset $(P \cup Q,<)$ where $x<y$ if and only if $\left(x, y \in P\right.$ and $\left.x<_{P} y\right)$ or $\left(x, y \in Q\right.$ and $\left.x<_{Q} y\right)$. The sum (or sequential composition) $P+Q$ of $P$ and $Q$ is the poset $(P \cup Q,<)$ such that $x<y$ if and only if one of the following conditions is true:

$$
\begin{aligned}
& -x \in P, y \in P \text { and } x<_{P} y ; \\
& -x \in Q, y \in Q \text { and } x<_{Q} y
\end{aligned} \quad-x \in P \text { and } y \in Q \text {. }
$$

The sum of two posets can be generalized to any linearly ordered sequence of pairwise disjoint posets: if $J$ is a linear ordering and $\left(\left(P_{j},<_{j}\right)\right)_{j \in J}$ is a sequence of posets, then $\sum_{j \in J} P_{j}=\left(\cup_{j \in J} P_{j},<\right)$ such that $x<y$ if and only if $\left(x \in P_{j}\right.$, $y \in P_{j}$ and $\left.x<_{j} y\right)$ or $\left(x \in P_{j}\right.$ and $y \in P_{k}$ and $\left.j<k\right)$. The sequence $\left(\left(P_{j},<_{j}\right)\right)_{j \in J}$ is called a $J$-factorization, or factorization for short, of the poset $\sum_{j \in J} P_{j}$. The only poset $(\emptyset,<)$ of width 0 is called empty poset and is denoted by $\epsilon$. 
Definition 2. The class $S P^{\diamond}$ of series-parallel scattered and countable posets is the smallest class of posets containing the empty poset, the singleton and closed under finite parallel composition and sum indexed by countable scattered linear orderings.

The class $S P^{\diamond}$ has a nice characterization in terms of graph properties: $S P^{\diamond}$ coincides with the class of scattered and countable $N$-free posets without infinite antichain. A poset $P$ is $N$-free if it does not contain $N$ as a sub-poset, that is if it does not contain elements $p, q, r, s \in P$ such that the ordering relations between those four elements are precisely $p<r, q<r$ and $q<s$.

Theorem 1. [3] A poset belongs to $S P^{\diamond}$ if and only if it is $N$-free, countable and scattered, and without infinite antichain.

An alphabet is a set whose elements are called letters. In this paper, we use only finite alphabet, thus the term "finite" is omitted. A poset labelled by $A$ is a poset $(P,<)$ equipped with a labelling map $l: P \rightarrow A$ which associates a letter to any element of $P$. The notion of a labelled poset corresponds to the notion of pomset in the literature. Also, the finite labelled posets of width 1 correspond to the usual notion of words. In order to shorten the notation, we make no distinction between a poset and a labelled poset. The class of posets of $S P^{\diamond}$ labelled by $A$ is denoted by $S P^{\diamond}(A)$. A language of $S P^{\diamond}(A)$ is a subset of $S P^{\diamond}(A)$. The sequential and parallel composition of posets can naturally be extended to languages. If $L_{1}, L_{2} \subseteq S P^{\diamond}(A)$, then

$$
\begin{aligned}
L_{1} \cdot L_{2} & =\left\{P \in S P^{\diamond}(A): \exists P_{1} \in L_{1} \exists P_{2} \in L_{2} \text { such that } P=P_{1}+P_{2}\right\} \\
L_{1} \| L_{2} & =\left\{P \in S P^{\diamond}(A): \exists P_{1} \in L_{1} \exists P_{2} \in L_{2} \text { such that } P=P_{1} \| P_{2}\right\}
\end{aligned}
$$

Let $n$ be an integer. We denote by $S P_{<n}^{\diamond}(A)$ the set of posets of $S P^{\diamond}(A)$ of width at most $n$ and by $S P_{*}^{\diamond}(A)$ the class $S P^{\diamond}(A)$ restricted to posets of finite width. A language $L$ has bounded-width if there exists an integer $n$ such that $L$ contains only posets of width at most $n$.

We now focus on the definitions of algebras for the recognition of languages. A semigroup $(S, \cdot)$ is a set $S$ equipped with an associative binary operation . called product. A \|-semigroup $[14-16](S, \cdot, \|)$ is an algebra such that $(S, \cdot)$ is a semigroup and $(S, \|)$ is a commutative semigroup. In ambiguous contexts, the - and \| products are respectively called sequential (or series) and parallel. The $\diamond$-semigroups are a generalization of semigroups for the recognition of words indexed by countable and scattered linear orderings (see [8] for more details): a $\diamond$-semigroup $(S, \Pi)$ is a set equipped with a map $\prod$ (also called sequential product) which associates an element of $S$ to any countable and linearly ordered sequence $s=\left(s_{j}\right)_{j \in J}$ (with $J \in \mathcal{S}$ ) of elements of $S$, such that $\prod(t)=t$ for any element $t$ of $S$ and $\prod$ is associative (i.e. for any factorization of the sequence $s$ into a sequence of sequences $\left.\left(t_{j}\right)_{j \in J^{\prime}}, \prod(s)=\prod\left(\left(\prod t_{j}\right)_{j \in J^{\prime}}\right)\right)$. Finally, a $\|-\diamond-$ semigroup $(S, \Pi, \|)$ is an algebra such that $(S, \Pi)$ and $(S, \|)$ are respectively a $\diamond-$ and a $\|$-semigroup. Recall that an algebra is finite if it is composed of a finite number of elements. Even if a $\|-\diamond$-semigroup is finite, the description of the 
product $\prod$ is not finite since the product of any sequence of countable length must be given. Actually, the sequential product of a finite $\|-\diamond$-semigroup can be described in a finite manner: we refer to [8] for this description in the case of finite $\diamond$-semigroups, which also immediately applies to finite $\|-\diamond$-semigroups. Even if the notion of a $\|-\diamond$-semigroup does not really fit into the general framework of universal algebra, the following notions are self understanding (we refer to [1] for precise definitions in the framework of universal algebra): sub-algebra, term, congruence, quotient, morphism between two algebras of the same type (i.e. two semigroups, or $\|$-semigroups, or $\diamond$-semigroups, or $\|-\diamond$-semigroups), free algebra, terms.

In order to lighten the notation we often denote an algebra by its set of elements: for example, we denote the semigroup $(S, \cdot)$ by $S$. We denote by $S^{1}$ the algebra $S$ if $S$ has an identity for all its operations, $S \cup\{1\}$ otherwise, 1 being an identity for all the operations. We also denote by $A^{+}, S P(A)$ [14-16] and $A^{\diamond}[8]$ respectively the free semigroup, $\|$-semigroup, and $\diamond$-semigroup over the set $A$. Let $S$ and $T$ be two algebras of the same type. Then $S$ divides $T$ if $S$ is the quotient of a sub-algebra of $T$. A morphism $\varphi: S \rightarrow T$ recognizes a subset $X$ of $S$ if $\varphi^{-1} \varphi(X)=X$. We say that $T$ recognizes $X$ if there exists a morphism from $S$ into $T$ recognizing $X$. A subset $X$ of an algebra $S$ is recognizable if there exist a finite algebra $T$ with the same type as $S$ and a morphism $\varphi: S \rightarrow T$ that recognizes $X$.

Proposition 1. Let $A$ be an alphabet. Then $S P^{\diamond}(A)$ is the free $\|-\diamond$-semigroup over A.

Example 2. Let $A=\{a, b\}$ and $L \subseteq S P_{\leq 2}^{\diamond}(A)$ be the language of non-empty posets $P$ such that $P$ has width at most 2 and each letter $a$ that appears into a parallel part of $P$ is incomparable with a $b$. Let $S=\{a, b, a b, p, 0,1\}$ be the finite $\|-\diamond$-semigroup defined by the following $\|$ commutative product: $a \| a=$ $a b\|a=0, p\| x=0$ for all $x \in S, a\|b=a b\| b=a b\|a b=b\| b=p$ and the sequential product $\prod$ such that, for any non-empty sequence $\left(s_{j}\right)_{j \in \mathcal{J}}$ $(J \in \mathcal{S}-\{\emptyset\})$ of elements of $S$,

$$
\prod\left(\left(s_{j}\right)_{j \in J}\right)= \begin{cases}a & \text { if }\left(s_{j}\right)_{j \in J} \text { contains only as } \\ a b & \text { if }\left(s_{j}\right)_{j \in J} \text { contains at least one } a \text { and one } b \\ b & \text { if }\left(s_{j}\right)_{j \in J} \text { contains only } b \mathrm{~s} \\ p & \text { if }\left(s_{j}\right)_{j \in J} \text { contains only } p, a, b, a b, \text { with at least one } p\end{cases}
$$

The elements 1 and 0 are respectively neutral and a zero for both $\prod$ and $\|$. Let $\varphi: S P^{\diamond}(A) \rightarrow S$ be the morphism defined by $\varphi(a)=a$ and $\varphi(b)=b$. Then $L=\varphi^{-1}(\{a, b, a b, p\})$.

\section{Series-rational languages}

Let $A$ be an alphabet. Let $L$ and $L^{\prime}$ be bounded-width languages of $S P^{\diamond}(A)$. The following operations are used in order to form the series-rational expressions 
for bounded-width languages of labelled posets:

$$
\begin{aligned}
L^{*} & =\left\{\sum_{j \in n} P_{j} \mid n \in \mathcal{N}, P_{j} \in L\right\} \\
L^{\omega} & =\left\{\sum_{j \in \omega} P_{j} \mid P_{j} \in L\right\} \\
L^{-\omega} & =\left\{\sum_{j \in-\omega} P_{j} \mid P_{j} \in L\right\} \\
L^{\natural} & =\left\{\sum_{j \in \alpha} P_{j} \mid \alpha \in \mathcal{O}, \alpha \neq 0, P_{j} \in L\right\} \\
L^{-\natural} & =\left\{\sum_{j \in-\alpha} P_{j} \mid \alpha \in \mathcal{O}, \alpha \neq 0, P_{j} \in L\right\} \\
L_{1} \diamond L_{2} & =\left\{\sum_{j \in J \cup \hat{J}^{*}} P_{j} \mid J \in \mathcal{S}-\{\emptyset\}, P_{j} \in L_{1} \text { if } j \in J \text { and } P_{j} \in L_{2} \text { if } j \in \hat{J}^{*}\right\}
\end{aligned}
$$

The class of series-rational languages over an alphabet $A$ is the smallest containing $\epsilon,\{a\}$ for all $a \in A$, and closed by finite union, finite sequential and parallel compositions, finite sequential iteration ${ }^{*}, \omega$ and $-\omega$-iterations, iteration on ordinals $\sharp$ and reverse iteration on ordinals $-\downarrow$ as well as diamond operator $\diamond$. We denote by $L(e)$ the language described by a series-rational expression $e$. If $E \subseteq S P^{\diamond}(A)$, then $E^{\diamond}$ is an abbreviation for $E \diamond \epsilon$. Note that if $\epsilon \notin E$, then $\epsilon \notin E^{\diamond}$.

A language is linear-rational if it is series-rational without using the $\|$ operator. Note that the linear-rational expressions are precisely those of Bruyère and Carton [5] over words on scattered and countable linear orderings. The following Theorem is a reformulation of a result of Carton and Rispal on recognizable languages of words on scattered and countable linear orderings.

Theorem 2. [8] Let $A$ be an alphabet, and $L$ be a language of $S P_{\leq 1}^{\diamond}(A)$. Then $L$ is linear-rational iff it is recognizable.

\section{Algebra}

We now define a pre-ordering relation $\prec_{\|}$, on the elements of a $\|-\diamond$-semigroup $S$, in order to adapt the notion of the depth of an element of a $s p$-algebra [14] to \| $-\diamond$-semigroups. If $s, t \in S$, then $s \prec t$ if and only if there exist $x_{1}, x_{2}, x_{3}, x_{4}, x_{5} \in$ $S^{1}$ such that $s=x_{1}\left(x_{2} \|\left(x_{3} t x_{4}\right)\right) x_{5}$ and $x_{2} \|\left(x_{3} t x_{4}\right) \neq x_{3} t x_{4}$. The relations $\prec_{\|}$and $\preceq_{\|}$are respectively the transitive and reflexive-transitive closures of $\prec$. Observe that if $S$ has a zero 0 or an identity 1 for all its operations, then the last condition of the definition of $\prec$ ensures that $0 \nprec_{\|} 0$ and $1 \nprec_{\|} 1$. By construction, $\preceq_{\|}$is a pre-order on $S$. A $\|-\diamond$-semigroup $S$ is depth-graded if, for each $s \in S$, there exists an integer $n$ such that each $\prec_{\|}$-chain with $s$ as least element has length at most $n$. In a depth-graded algebra, the relation $\prec_{\|}$ 
is irreflexive, and hence is a strict partial order. The depth of $s$, denoted $d p(s)$, is defined inductively on the elements of a depth-graded $\|-\diamond$-semigroup $S$ by

$d p(s)= \begin{cases}1 & \text { if there does not exist } t \in S \text { such that } s \prec_{\|} t \\ 1+\sup \left\{d p(t): s \prec_{\|} t\right\} & \text { otherwise. }\end{cases}$

A $\|-\diamond$-semigroup $S$ has bounded depth if there exists an integer $n$ such that each $\prec_{\|}$-chain is of length at most $n$. Observe that if $S$ is bounded-depth, then it is also depth-graded.

Example 3. The poset of Figure 3 has depth 4 and width 6 . Observe that $S P^{\diamond}(A)$

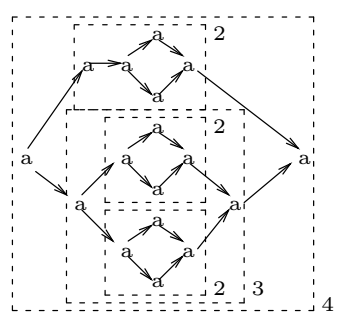

Fig. 1. A finite poset of depth 4 . The depth of some sub-posets into frames is indicated.

is not depth-graded. Consider for example the poset $P=\sum_{i<\omega} P_{i}$, where $P_{i}$ consists in $i$ copies of the letter $a$, all set in parallel, for each $i<\omega$. Then $P \in S P^{\diamond}(A)$, and $d p(P)$ is infinite. Observe that any poset of $S P_{*}^{\diamond}(A)$ has finite depth, and $S P_{*}^{\diamond}(A)$ is depth-graded. However, $S P_{*}^{\diamond}(A)$ does not have boundeddepth.

A $\|-\diamond$-semigroup $S$ is depth-nilpotent if it has bounded-depth and $S-\{1\}$ contains a 0 which is the only idempotent for the $\|$ operation. The following Lemma is an immediate adaptation of Lemma 3.5 of [15] to $\|-\diamond$-semigroups:

Lemma 1. Let $S$ be a $\|-\diamond$-semigroup with bounded-depth. Then $S$ is depthnilpotent iff for any $s, t \in S-\{1\}$ with $t \neq 0$, then $s \| t \neq t$.

\section{$5 \quad$ Logic}

The monadic second-order $(\mathrm{MSO}[<])$ logic is classical in set theory, and was first set up by Büchi for words. In this paper the formulæ of $\mathrm{MSO}[<]$ are interpreted over posets of $S P^{\diamond}(A)$, labelled by the letter of the alphabet $A$. Formal logic is used to specify a language by properties of its labelled posets, as for example "every antichain $X$ of a poset of the language such that $X$ has at least two elements contains at least two elements labelled by $a$ ". We now outline the basic notions on $\mathrm{MSO}[<]$. We refer e.g. to $[9,21]$ for more details. 
We first focus on first-order logic. The first order variables are named by lower-cases letters like $x, y, z$ and are interpreted over elements of the posets. An existential $(\exists)$ or an universal $(\forall)$ quantification can be applied to a variable: in this case, the variable is said to be bounded to the quantifier. An unbounded variable is free. The basic ingredients for formulæ construction are the atomic formula. If $x, y$ and $a$ are respectively two first-order variables and a letter of the alphabet, then $R_{a}(x)$ and $x<y$ are atomic formulæ: the first one meaning that $x$ is associated to an element labelled by $a$ and the other is self-understanding. The logical symbols $\wedge, \vee$ and $\neg$ are the usual connectives. Parenthesis ensure legibility of formulæ. We write $\varphi\left(x_{1}, \ldots, x_{n}\right)$ to denote that $\varphi$ has at most $x_{1}, \ldots, x_{n}$ as free variables. A sentence is a formula without free variable.

The satisfaction relation $\models$ between series-parallel labelled posets $(P,<)$ and logic formulæ is defined canonically. Let $\phi\left(x_{1}, \ldots, x_{n}\right)$ be a formula having at most $x_{1}, \ldots, x_{n}$ as free variables, $(P,<)$ be a poset and $p_{1}, \ldots, p_{n} \in P$. Then $(P,<), p_{1}, \ldots, p_{n} \models \phi\left(x_{1}, \ldots, x_{n}\right)$ means that $(P,<)$ satisfies $\varphi$ when $p_{1}, \ldots, p_{n}$ serve as respective interpretations for $x_{1}, \ldots, x_{n}$.

We define the second-order logic $\mathrm{MSO}[<]$ as an extension of the first-order logic. In $\mathrm{MSO}[<]$, variables that range over sets of elements of posets are also allowed in addition to first-order variables. We use upper-cases letters like $X, Y, Z$ to name these variables, called second-order variables. Comparatively to firstorder logic, $\mathrm{MSO}[<]$ has one more form of atomic formula, which is self-understanding: $x \in X$, where $x$ and $X$ are respectively a first and a second-order variable. The notions and notations introduced for first-order logic can naturally be extended to second-order logic.

The language $L(\phi)$ of a sentence $\phi$ is the set of all labelled-posets satisfying $\phi$. A property $p$ is definable in $\mathrm{MSO}[<]$ if there exists a formula of $\mathrm{MSO}[<]$ that expresses $p$.

Let $n$ be an integer, $A$ an alphabet, $P$ and $P^{\prime}$ two labelled posets. Following the notation from [9], we write $P \equiv_{n} P^{\prime}$ if $P$ and $P^{\prime}$ satisfy the same sentences of quantifier rank $\leq n$ (the quantifier rank is the maximum number of nested quantifiers in a formula). It is a well-known result that $\equiv_{n}$ is an equivalence relation with finitely many equivalence classes. Furthermore, $S P(A) / \equiv_{n}$ is a finite ||-semigroup (see for example Proposition 3.1.4 of [9]). This is only verification to check, using the same arguments as in the proof of Proposition 3.1.4 of [9], that

Proposition 2. Let $A$ be an alphabet and $n$ an integer. Then $S P^{\diamond}(A) / \equiv_{n}$ (resp. $A^{\diamond} / \equiv_{n}$ ) is a finite $\|-\diamond$-semigroup (resp. $\diamond$-semigroup) that recognizes $L(\phi)$ for any sentence $\phi$ of quantifier rank $\leq n$.

In order to enhance readability of formulæ we use several notations and abbreviations for properties expressible in $\mathrm{MSO}[<]$. The following are usual and self-understanding: $\phi \rightarrow \psi, X \subseteq Y, x=y$. We denote "there exists an unique $x$ " by $\exists ! x$, " $x$ and $y$ are different and not comparable" by $x \| y$, "there exists a non-empty set $X$ " by $\bar{\exists} X$, "set $X$ has cardinality $j$ " by $\operatorname{Card}_{j}(X)$, where $j$ is any integer, "set $X$ is an antichain" by Antichain $(X)$, "sets $U$ and $V$ form a partition of $X$ " by Partition $(U, V, X)$. All those properties are definable in 
$\operatorname{MSO}[<]$. A set $X$ is finite iff every non-empty subset of $X$ has a minimum and a maximum. It is isomorphic to $\omega$ (resp. $-\omega$ ) if it is linearly ordered, infinite and every part of $X$ with a maximum (resp. minimum) is finite. Again, all the properties above are definable in $\mathrm{MSO}[<]$. A simple transcription of Definition 1 into $\mathrm{MSO}[<]$ gives a monadic second-order formula that tests if an ordered set is a complete linear ordering. Finally, we need the following abbreviation:

$$
X=U \oplus V \equiv \operatorname{Partition}(U, V, X) \wedge(\forall u \forall v u \in U \wedge v \in V \rightarrow \neg u \| v)
$$

Example 4. Let $A=\{a, b\}$ be an alphabet and $L \subseteq S P^{\diamond}(A)$ be the language of posets verifying the property "every antichain $X$ of a poset of the language such that $X$ has at least two elements contains at least two elements labelled by $a$ ". Let $\phi$ be the following sentence of $\operatorname{MSO}[<]$. Then $L(\phi)=L$.

$$
\begin{array}{r}
\phi \equiv \forall X \text { Antichain }(X) \rightarrow \exists Y Y \subseteq X \wedge \operatorname{Card}_{2}(Y) \rightarrow \\
\left(\exists x \exists y x \in X \wedge y \in X \wedge x \neq y \wedge R_{a}(x) \wedge R_{a}(y)\right)
\end{array}
$$

\section{Equivalence}

This section is devoted to the main result of this paper:

Theorem 3. Let $A$ be an alphabet and $L \subseteq S P^{\diamond}(A)$. Then the following assertions are equivalent:

1. L is series-rational,

2. there exists a morphism $\varphi: S P^{\diamond}(A) \rightarrow S$ into a finite depth-nilpotent $\|-\diamond$ semigroup $S$ and $X \subseteq S$ such that $0 \notin X, L=\varphi^{-1}(X)$, and $\varphi(a) \neq 1$ for all $a \in A$,

3. $L$ has bounded-width and is definable by a sentence of $M S O[<]$.

Example 5. Let $A=\{a, b\}$ and $L$ be the language of Example 2. Let $X \subseteq$ $S P^{\diamond}(A)$ : for convenience we define the abbreviation $X^{\diamond 1}$ for $X^{\diamond} \cup\{\epsilon\}$. Then $L$ is the language of the series-rational expression

$$
\begin{aligned}
e & =\left(A^{\diamond 1} a A^{\diamond 1}\left\|b^{\diamond}+e^{\prime}\right\| e^{\prime}+A^{\diamond}+b^{\diamond} \| b^{\diamond}\right)^{\diamond} \\
e^{\prime} & =A^{\diamond 1} a A^{\diamond 1} b A^{\diamond 1}+A^{\diamond 1} b A^{\diamond 1} a A^{\diamond 1}
\end{aligned}
$$

and of the logical sentence

$$
\phi_{e} \equiv \phi_{\mathrm{wd} \in\{1,2\}} \wedge \forall x\left(R_{a}(x) \wedge \neg \mathrm{Flat}(x)\right) \rightarrow \exists y x \| y \wedge R_{b}(y)
$$

where $\phi_{\mathrm{wd} \in\{1,2\}} \equiv \forall X \operatorname{Antichain}(X) \rightarrow \vee_{1 \leq i \leq 2} \operatorname{Card}_{i}(X)$ and Flat $(x) \equiv \forall y x=$ $y \vee x<y \vee y<x$. Furthermore, it can be easily checked that the morphism of $\|-\diamond$-semigroups of Example 2, that recognizes $L$, also verifies the properties of Theorem 3. 
Observe that the language $L$ of Example 4 is definable in $\operatorname{MSO}[<]$, but as it has not bounded width it is not series-rational. It is also recognizable by a morphism $\varphi: S P^{\diamond}(A) \rightarrow S$, with $S$ finite and depth-nilpotent, but it can be easily checked by the reader that in this case $0 \in \varphi(L)$.

In the remainder of this section we will give a sketch of the proof of Theorem 3. Proposition 3 shows that 2 implies 1, and Proposition 4 that 3 implies 2. The proof of Proposition 3 essentially relies on an induction over the depth of $s \in S-\{0\}$. Theorem 2 solves the induction step $d p(s)=1$. Proposition 4 relies on Proposition 2, whose proof uses classical Ehrenfeucht-Fraïssé games arguments.

Proposition 3. Let $A$ be an alphabet, $S$ be a finite depth-nilpotent $\|-\diamond$-semigroup and $\varphi: S P^{\diamond}(A) \rightarrow S$ a morphism that recognizes $L \subseteq S P^{\diamond}(A)$, such that $0 \notin \varphi(L)$ and $\varphi(a) \neq 1$ for all $a \in A$. Then $L$ is series-rational.

Proposition 4. Let $A$ be an alphabet, $\phi$ a monadic second-order sentence and $m$ an integer. Let $L=L(\phi) \cap S P_{\leq m}^{\diamond}(A)$. There exist a morphism $\varphi: S P^{\diamond}(A) \rightarrow S$

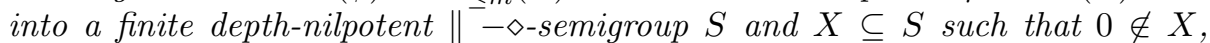
$L=\varphi^{-1}(X)$, and $\varphi(a) \neq 1$ for all $a \in A$.

As a series-rational language has necessarily bounded-width, it remains to show that it can also be defined using a sentence of $\operatorname{MSO}[<]$. We now give a sketch of the proof, which proceeds by induction on a series-rational expression $e$, by extension of the ideas from [17]. For simplicity we do not consider the empty poset in the discussion. Let $P$ be a non-trivial poset such that $P \in L(e)$. Then by definition $P$ is obtained from others posets of $S P^{\diamond}(A)$ using the seriesrational operators. Let $Q$ be such a non-empty subset of $P$. Then $Q$ verifies two properties. First, any element of $P$ between two comparable elements of $Q$ also belongs to $Q$ : we say that $Q$ has the block property. Second, if an element $x$ of $P$ is comparable with an element of $Q$ and incomparable with another one, then $x$ belongs to $Q$ : we say that $Q$ has the good part property. Those two properties are definable in $\mathrm{MSO}[<]$. Let $C$ be a non-empty good part of $P$ which can be decomposed into an union of maximal (with respect to inclusion) blocks. If such a decomposition exists it is unique. We construct, by induction on $e$, a formula $\varphi_{e}(X)$ with exactly one free variable $X$, which is second-order, and such that $P, C \models \varphi_{e}(X)$ iff $D \in L(e)$ for each maximal block $D$ of $C$. The basic step of the induction, where $e$ is a letter, is trivial. The induction step where $e$ has the form $e_{1} \| e_{2}$ (resp. $\left.e_{1} \cdot e_{2}\right)$ is easy: it suffices to express in $\mathrm{MSO}[<]$ that each maximal block $D$ of $C$ can be partitioned into $U$ and $V$ such that all the elements of $U$ are incomparable with (resp. less than) all the elements of $V$. As it can be verified that $U$ and $V$ are good parts of $C$, the induction hypothesis applied on $U, V, e_{1}$ and $e_{2}$ permits to conclude. The other cases $\left(^{*}, \omega,-\omega,{ }^{\natural},-{ }^{\natural}\right.$ and $\diamond)$ use extensions of this principle. Let us focus on the case $e=e^{\prime \omega}$ for example, the other induction steps being similar. Let $D$ be a maximal block of $C$. Then $D \in L(e)$ iff there exist $J \in \mathcal{S}$ and a factorization of $D$ into a sum of posets $D=\sum_{j \in J} D_{j}$, such that $J$ is isomorphic to $\omega$ and $D_{j} \in L\left(e^{\prime}\right)$ for each $j \in J$. The linear ordering $J$ can be partitioned itself into $J_{1}$ and $J_{2}$ such that 
if $x \in J_{i}$ then $x+1 \in J_{k}$ for any $x \in J$, where $i, k \in\{1,2\}$ and $i \neq k$. Thus, $D$ can be partitioned into $U$ and $V$ such that $x \in U$ (resp. $x \in V$ ) iff $x \in D_{j}$ for some $j \in J_{1}$ (resp. $j \in J_{2}$ ). Finally, $D \in L(e)$ iff there exist $U$ and $V$ such that $D=U \oplus V$, each maximal (with respect to inclusion) block of $U$ and $V$ is a $D_{j}$ for some $j \in J$, and $J$ is isomorphic to $\omega$. As it can be verified that each maximal block of $U$ or $V$ is a good part of $P$, the induction hypothesis can be applied. Figure 2 illustrates such a factorization of $D$. The formula $\varphi_{e^{\prime \omega}}(X)$ is

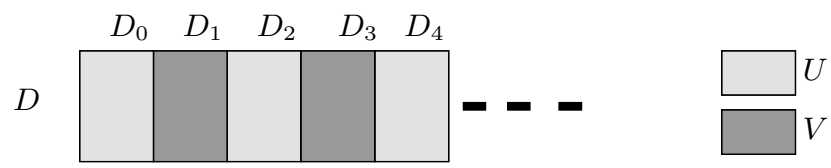

Fig. 2. A poset $D \in S P^{\diamond}(A)$ belongs to $L\left(e^{\prime \omega}\right)$ iff it can be decomposed into $D=$ $\sum_{j \in \omega} D_{j}$ where $D_{j} \in L\left(e^{\prime}\right)$ for all $j \in \omega$. Following this decomposition, $D \in L\left(e^{\prime \omega}\right)$ iff it can be decomposed into $D=U \oplus V$, where each maximal block of $U$ or $V$ is a $D_{j}$ for some $j \in \omega$. A linear ordering $W$ isomorphic to $\omega$ can be built by taking one element in each maximal block of $U$ and $V$.

given below:

$$
\begin{aligned}
\varphi_{e^{\prime \omega}}(X) & \equiv \forall Z \operatorname{MaxBlock}(Z, X) \rightarrow \bar{\exists} U \bar{\exists} V Z=U \oplus V \wedge \varphi_{e_{1}}(U) \\
& \wedge \varphi_{e_{1}}(V) \wedge(\forall W \operatorname{Ordertype}(W, U, V) \rightarrow \operatorname{Omega}(W)) \\
\operatorname{Ordertype}(Z, X, Y) & \equiv Z \subseteq X \cup Y \wedge \forall B \operatorname{MaxBlock}(B, X) \rightarrow \exists ! z z \in Z \cap B \\
& \wedge \forall B \operatorname{MaxBlock}(B, Y) \rightarrow \exists ! z z \in Z \cap B
\end{aligned}
$$

For the other iteration operators, it suffices to change the test on $W$ at the end of the formula $\varphi_{e^{\prime \omega}}$. For example, if the iteration operator is ${ }^{\natural}$, Omega $(W)$ is changed in order to check that $W$ does not contain $-\omega$ as sub-ordering. The $\diamond$ composition of languages is processed using very similar arguments, and relies on Lemma 2, which gives another definition of $\diamond$, more adapted to a translation in the formalism of $\mathrm{MSO}[<]$ than the one of Section 3.

Lemma 2. Let $A$ be an alphabet and $L_{1}$ and $L_{2}$ be two languages of $S P^{\diamond}(A)$. Then $P \in L_{1} \diamond L_{2}$ if and only if there exist a scattered and countable linear ordering $K \neq \emptyset$, a sequence $\left(P_{k}\right)_{k \in K}$ of posets of $S P^{\diamond}(A)$ and a map $f: K \rightarrow$ $\{1,2\}$ such that the following conditions are true:

1. $P=\sum_{k \in K} P_{k}$;

2. if $f(k)=i$ and $k+1 \in K$ then $f(k+1) \neq i$;

3. if $k \in K, k$ is not the last element of $K$, and $k$ has no successor, then $f(k)=2$;

4. if $k \in K, k$ is not the first element of $K$, and $k$ has no predecessor, then $f(k)=2$;

$Z \subseteq X \cup Y$ has been added to cor-
rect a flaw in the published version of the paper. 
5. if $k$ is the first or the last element of $K$, then $f(k)=1$;

6. $K$ is complete;

7. $f(k)=i$ implies $P_{k} \in L_{i}$.

In order to conclude, it suffices to observe that $P$ is a good part and a maximal block of itself. Let $\psi_{e} \equiv \exists X(\forall x x \in X) \wedge \varphi_{e}(X)$. Then $L(e)=L\left(\psi_{e}\right)$. As a consequence:

Proposition 5. Let $A$ be an alphabet and $L \subseteq S P^{\diamond}(A)$. If $L$ is series-rational, then $L=L\left(\psi_{e}\right)$ for any series-rational expression e such that $L=L(e)$.

We finish by providing a last example.

Example 6. Let $A=\{a, b\}$ and $L \subseteq S P^{\diamond}(A)$ be the language of posets $P$ of width at most 2 , such that, if a $a$ appears in a parallel part $P_{1} \| P_{2}$ of $P$, then $P_{1} \| P_{2}$ is immediately followed by $P_{3}$ such that $w d\left(P_{3}\right)=1$ and $P_{3}$ contains a $b$. Let $e_{1}=\left(A+\left(b^{\diamond} \| b^{\diamond}\right)\right)^{\diamond}+\epsilon$. The language of $e_{1}$ is all the posets that do not contain an $a$ in parallel with another letter. Then $L$ is the language of the series-rational expression

$$
e=\left(e_{1}\left(\left(\left(A^{\diamond}+\epsilon\right) a\left(A^{\diamond}+\epsilon\right)\right) \| A^{\diamond}\right)\left(A^{\diamond}+\epsilon\right) b\right)^{\diamond} e_{1}+e_{1}
$$

and of the second-order monadic sentence interpreted over $S P^{\diamond}(A)$

$$
\begin{aligned}
& \phi_{e} \equiv \phi_{\leq 2} \wedge \forall x \forall y\left(R_{a}(x) \wedge x \| y\right) \rightarrow \\
& \quad \exists z x<z \wedge R_{b}(z) \wedge \operatorname{Flat}(z) \wedge \forall w x<w<z \rightarrow \operatorname{Flat}(w) \vee w \| y
\end{aligned}
$$

where $\phi_{\leq 2} \equiv \forall X$ Antichain $(X) \rightarrow \vee_{i \leq 2} \operatorname{Card}_{i}(X)$ and Flat $(x) \equiv \forall y x=y \vee x<$ $y \vee y<x$.

We now turn to the definition of a finite $\|-\diamond$-semigroup recognizing $L$. Even when a $\|-\diamond$-semigroup $S$ has a finite number of elements, its description is infinite because the result of the sequential product of any sequence of element of $S$ must be given, and there are an infinity of them. A consequence is that finite $\|-\diamond$-semigroups do not really fit in the general framework of universal algebra. However, when the cardinal of $S$ is finite, the sequential product can equivalently be replaced by three operations of finite arity: the sequential product of two elements, the $\omega$ repetition of an element and the reverse $\omega$ repetition. Formally, let $x \in S$ :

$$
\begin{aligned}
x^{\omega} & =\prod\left(s_{i}\right)_{i \in \omega} \text { where } s_{i}=x \text { for all } i \in \omega \\
x^{-\omega} & =\prod\left(s_{i}\right)_{i \in-\omega} \text { where } s_{i}=x \text { for all } i \in-\omega
\end{aligned}
$$

This technique was introduced by Wilke [24] in the case of finite $\omega$-semigroups, and applied to the case of $\diamond$-semigroups in [8]. We use it in this example.

Let $S=\{a, b, a b, p, q, r, 0,1\}$ be the finite $\|-\diamond$-semigroup defined by $a \| a=$ $a\|b=a\| a b=a b\|b=a b\| a b=p, b \| b=(a \| b) b=q, b(b \| b)=b(a \| b) b=$ $r, b(a \| b)=s, p\|x=q\| x=r \| x=0$ for all $x \in S \quad$ and by the binary sequential product, $\omega$ and $-\omega$ operations of Figure 3. It can easily be checked that $S$ is depth-nilpotent. Let $\varphi: S P^{\diamond}(A) \rightarrow S$ be the morphism defined by $\varphi(a)=a$ and $\varphi(b)=b$. Then $L=\varphi^{-1}(\{a, b, a b, q, r, 1\})$. 

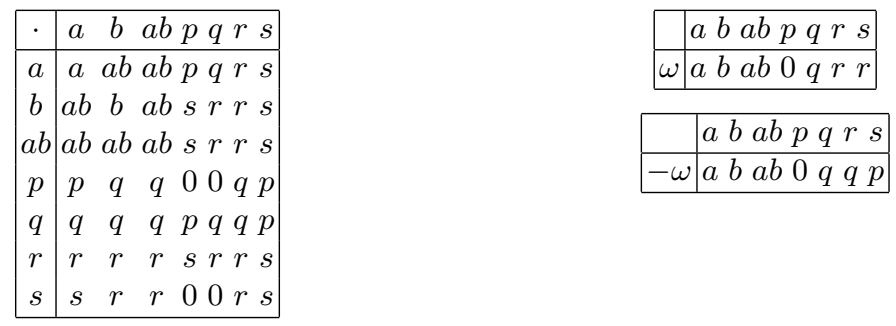

Fig. 3. A finite description of the sequential product of $S$.

\section{Conclusion}

The proof of the main result of the paper is effective. A consequence is that MSO $[<]$ interpreted over $S P_{\leq n}^{\diamond}$ is decidable. As a corollary, any question on series-rational languages expressible in $\mathrm{MSO}[<]$, as for example the inclusion problem of series-rational languages, is also decidable.

Linear-rational expressions were introduced in [5] as well as equivalent automata. It is known from [2] that a language of scattered and countable linear structures is linear-rational if and only if it is in $\mathrm{MSO}[<]$. When non-scattered linear structures are taken into account, a shuffle operation [4] must be added to linear-rational expressions to keep the equivalence with automata. Linearrational languages with shuffle are a strict subset of the languages of $\mathrm{MSO}[<]$ interpreted over all countable linear structures.

A notion of automata accepting labelled posets of $S P^{\diamond}(A)$ have been introduced in [3], as well as equivalent rational expressions, called series-parallel rational expressions. The series-parallel rational expressions are an extension of the series-rational expressions, and automata equivalent to the latter, called fork-acyclic, are a restriction of automata of the former. The link between seriesparallel rationality, series-rationality, automata and logic was first studied by Lodaya and Weil [14] and Kuske [11] for finite and $\omega$ structures. In this case, second-order definability coincides with series-rationality. One more assertion could be added to Theorem 3 :

Theorem 4. Let $A$ be an alphabet and $L \subseteq S P^{\diamond}(A)$. Then the following assertions are equivalent:

1. L is series-rational,

2. there exists a morphism $\varphi: S P^{\diamond}(A) \rightarrow S$ into a finite depth-nilpotent $\|-\diamond$ semigroup $S$ and $X \subseteq S$ such that $0 \notin X, L=\varphi^{-1}(X)$, and $\varphi(a) \neq 1$ for all $a \in A$,

3. $L$ has bounded-width and is definable by a sentence of $M S O[<]$,

4. $L$ is accepted by a fork-acyclic automaton.

Finite $\|-\diamond$-semigroups have important properties. One of them was used in Example 6: the sequential product can be equivalently replaced by operations 
whose descriptions are finite. This makes finite $\|-\diamond$-semigroups really finite algebraic objects. As another property, a particular finite $\|-\diamond$-semigroup can be canonically attached to any recognizable language $L$ of posets of $S P^{\diamond}(A)$. This finite $\|-\diamond$-semigroup, called the syntactic $\|-\diamond$-semigroup of $L$, divides any $\|-\diamond$-semigroup recognizing $L$. In formal languages theory, this algebra plays an important role. The importance of syntactic algebras is emphasized for infinite structures: for example, contrary to the finite words case, a minimal automaton can not any more be attached to a recognizable language of infinite words. The existence of the syntactic $\|-\diamond$-semigroup of a recognizable language of $S P^{\diamond}(A)$ lays the foundation for the generalization of numerous results of formal languages of infinite words.

Finally, let us mention the study of Ésik and Németh [10] on rational sets of finite series-parallel posets, but with a non-commutative parallel composition. In this case, as in the commutative parallel case, rationality, regularity, recognizability and logical definability all coincide for bounded-width languages.

\section{Acknowledgements}

The author wishes to express his thanks to the anonymous referees for helpful remarks that have been used to improve the quality of this paper.

\section{References}

1. Jorge Almeida. Finite semigroups and universal algebra, volume 3 of Series in algebra. World Scientific, 1994.

2. Nicolas Bedon, Alexis Bès, Olivier Carton, and Chloé Rispal. Logic and rational languages of words indexed by linear orderings. In Edward A. Hirsch, Alexander A. Razborov, Alexei Semenov, and Anatol Slissenko, editors, Computer Science in Russia 2008, 3rd International Symposium, Moscow, volume 5010 of Lect. Notes in Comput. Sci., pages 76-85. Springer-Verlag, June 2008.

3. Nicolas Bedon and Chloé Rispal. Series-parallel languages on scattered and countable posets. In Ludek Kucera and Antonín Kucera, editors, $M F C S^{\prime}$ '07, volume 4708 of Lect. Notes in Comput. Sci., pages 477-488. Springer-Verlag, August 2007.

4. Alexis Bès and Olivier Carton. A Kleene theorem for languages of words indexed by linear orderings. Int. J. Found. Comput. Sci., 17(3):519-542, 2006.

5. Véronique Bruyère and Olivier Carton. Automata on linear orderings. In M. Ito and M. Toyama, editors, DLT'2002, volume 2450 of Lect. Notes in Comput. Sci., pages 103-115. Springer-Verlag, 2002.

6. J. Richard Büchi. On a decision method in the restricted second-order arithmetic. In Proc. Intern. Congr. on Logic, Methodology and Philosophy of Science, Berkeley 1960, pages 1-11, Stanford, California, 1962. Stanford University Press.

7. J. Richard Büchi. Transfinite automata recursions and weak second order theory of ordinals. In Proc. Int. Congress Logic, Methodology, and Philosophy of Science, Jerusalem 1964, pages 2-23. North Holland, 1964.

8. Olivier Carton and Chloé Rispal. Complementation of rational sets on countable scattered linear orderings. Int. J. Found. Comput. Sci., 16(4):767-786, August 2005 . 
9. Heinz-Dieter Ebbinghaus and Jörg Flum. Finite model theory. Springer monographs in mathematics. Springer, 2nd edition, 1999.

10. Zoltán Ésik and Z.L. Németh. Automata on series-parallel biposets. In W. Kuich, G. Rozenberg, and A. Salomaa, editors, DLT'2001, volume 2295 of Lect. Notes in Comput. Sci., pages 217-227. Springer-Verlag, 2002.

11. Dietrich Kuske. Infinite series-parallel posets: logic and languages. In ICALP 2000, volume 1853 of Lect. Notes in Comput. Sci., pages 648-662. Springer-Verlag, 2000.

12. Dietrich Kuske. Towards a language theory for infinite N-free pomsets. Theoret. Comput. Sci., 299:347-386, 2003.

13. H. Laüchli and J. Leonard. On the elementary theory of linear order. Fund. Math., 59:109-116, 1966.

14. Kamal Lodaya and Pascal Weil. Series-parallel posets: algebra, automata and languages. In M. Morvan, Ch. Meinel, and D. Krob, editors, STACS'98, volume 1373 of Lect. Notes in Comput. Sci., pages 555-565. Springer-Verlag, 1998.

15. Kamal Lodaya and Pascal Weil. Series-parallel languages and the bounded-width property. Theoret. Comput. Sci., 237(1-2):347-380, 2000.

16. Kamal Lodaya and Pascal Weil. Rationality in algebras with a series operation. Inform. Comput., pages 269-293, 2001.

17. C. Michaux and F. Point. Les ensembles k-reconnaissables sont définissables dans $<N,+, V_{k}>$. (the k-recognizable sets are definable in $<N,+, V_{k}>$ ). C. R. Acad. Sci. Paris, Sér. I(303):939-942, 1986.

18. Michael Ozer Rabin. Decidability of second-order theories and automata on infinite trees. Trans. Amer. Math. Soc., 141:1-35, 1969.

19. Joseph G. Rosenstein. Linear Orderings. Academic Press, 1982.

20. Saharon Shelah. The monadic theory of order. Annals of Mathematics, 102(3):379419, November 1975.

21. Wolfgang Thomas. Languages, automata, and logic. In G. Rozenberg and A. Salomaa, editors, Handbook of Formal Languages, volume III, pages 389-455. SpringerVerlag, 1997.

22. Jacobo Valdes. Parsing flowcharts and series-parallel graphs. Technical Report STAN-CS-78-682, Computer science departement of the Stanford University, Standford, Ca., 1978.

23. Jacobo Valdes, Robert E. Tarjan, and Eugene L. Lawler. The recognition of series parallel digraphs. SIAM J. Comput., 11:298-313, 1982.

24. Thomas Wilke. An Eilenberg theorem for $\infty$-languages. In Automata, Languages and Programming: Proc. of 18th ICALP Conference, pages 588-599. SpringerVerlag, 1991. 